\title{
Extrapolation and Structural Similarity in Games*
}

\author{
Friederike Mengel ${ }^{\dagger} \ddagger$ \\ University of Essex \\ and Maastricht University
}

Emanuela Sciubba ${ }^{\S}$

Birkbeck, University of London

September 23, 2014

\begin{abstract}
We conduct a laboratory experiment and provide evidence of learning spillovers within and across equivalence classes of "structurally similar" games. These spillovers are inconsistent with existing theories of learning in games.
\end{abstract}

Keywords: Game Theory, Learning, Extrapolation, Similarity, Experiments.

JEL-classification: C72, C91.

${ }^{*}$ We thank Larry Blume, Ben Greiner, Frank Heinemann, Willemien Kets, Dan Levin, Joshua Miller, Erik Mohlin, Robert Sugden and an anonymous referee as well as seminar participants in East Anglia, Kopenhagen, Maastricht, Oslo, Santa Fe, Tilburg and Toulouse for helpful comments. Friederike Mengel thanks the European Union for financial support (grant PIEF2009-235973 and PERG08-GA-2010-277026). A previous version of this paper was circulated under the title "Extrapolation in Games of Coordination and Dominance Solvable Games".

${ }^{\dagger}$ Department of Economics, University of Essex, Wivenhoe Park, Colchster CO4 3SQ, United Kingdom, e-mail: fr.mengel@gmail.com

${ }^{\ddagger}$ Department of Economics (AE1), Maastricht University, PO Box 616, 6200 MD Maastricht, The Netherlands

${ }^{\S}$ Department of Economics, Mathematics and Statistics, Birkbeck, University of London, Malet Street, London WC1E 7HX. e.sciubba@bbk.ac.uk 


\section{Introduction}

How economic agents learn to make decisions in games has received a lot of attention by economists. In many cases of interest agents learn across several different strategic situations, and the number of possibilities is so vast that a particular situation is practically never experienced twice. A tacit assumption of standard learning models is that players extrapolate their experience from previous interactions similar to the current one. Which situations people perceive as similar and how they transfer knowledge between "similar" situations, though, has received much less attention in the literature. ${ }^{1}$

In this paper we study learning spillovers across equivalence classes of "structurally similar" games. ${ }^{2}$ We consider two classes of games: games which are solvable through deliberative reasoning and, more precisely, through iterated elimination of dominated strategies (henceforth IEDS) and coordination games where, unlike IEDS games, deductive equilibrium analysis based on common knowledge of rationality alone fails to determine a unique equilibrium strategy profile. Using those classes of games we can ask whether the concepts of dominance solvability and coordination that we use in game theory might be recognizable dimensions of similarity in the minds of participants or might at least be reflected in their choices.

We use a 3x2 design. In most treatments they play a sequence of two games: first 5 repetitions of a variant of the so called 'guessing game' (either the IEDS version or the original coordination version (Keynes,1936)) and afterwards ten repetitions of a 3x3 game, which is either an IEDS or coordination game. In our control treatments participants play only the $3 \times 3$ normal form games.

We find evidence of learning spillovers within and across equivalence classes of "structurally similar" games. Having played a structurally similar game before leads to faster convergence to Nash equilibrium (or coordination) in the $3 \times 3$ game. On the other hand playing a structurally different game leads to less Nash play (coordination), not only when compared to the situation where a similar game is played but also compared to the baseline, where no other game is played before. These treatment differences are not easily accommodated by standard models of learning in games, such as reinforcement learning, imitation or best response learning.

There are few other papers in the experimental literature which look at learning transfers, extrapolation or categorization. Haruvy and Stahl (2009) study learning transfer between 'dissimilar' symmetric normal form games. ${ }^{3}$ They find that a model of experience-weighted attraction learning augmented with action relabeling performs well in explaining the initial choices in each game. Grimm and Mengel (2012) study learning in a multiple games environment, where participants face different normal form games randomly drawn in each period. They demonstrate that learning spillovers occur whenever the complexity of the environment increases. One of their findings is that people learn to treat strategically equivalent games in the same way. Huck, Jehiel and Rutter (2011) also find evidence for categorical thinking based on belief learning in an experiment and Cason et al (2012) find evidence for behavioral spillovers in Coordination games. Several studies have found implicit evidence that there is learning transfer across games. Examples include

\footnotetext{
${ }^{1}$ For some theoretical work on this question see Gilboa and Schmeidler (1995), Jehiel (2005), Steiner and Stewart (2008) or Mengel (2012). Experimental literature will be discussed below in detail.

${ }^{2} \mathrm{~A}$ formal definition of structural equivalence classes of normal form games is provided by e.g. Germano (2006). Equivalence classes of games are defined by means of (i) discontinuities of the best response correspondence and (ii) relabeling of strategies and/or players.

${ }^{3}$ For them the term dissimilar means that there is no re-labeling of actions which makes games monotonic transformations of each other. This is true for all the games considered here, including those that we call 'structurally similar'. See also Rankin et al (2000).
} 
Rapoport et al. (2000), Cooper and Kagel (2003, 2008) or Chong et al. (2006) among others. Camerer et al (1998) study learning transfers across IEDS guessing games with different parameters. Interestingly, Kuo et al (2009) find that different brain regions are involved when participants play games that can be solved via deliberative reasoning (such as dominance solvable games) as opposed to coordination games. ${ }^{4}$

The paper is organized as follows. In Section 2 we describe the experimental design. In Section 3 we present the results. Section 4 concludes.

\section{Design}

336 students participated in one of the following treatments. In treatment $I E D S^{3 x 3}\left(C O R^{3 x 3}\right)$ participants were randomly matched in pairs for ten rounds to play the $3 \times 3$ game depicted on the left (right) in Table 1. In treatment IEDS- $I E D S^{3 x 3}$ participants were first matched in fixed groups of four to play the $70 \%$ (IEDS) version of the guessing game over five rounds. In this game all group members have to simultaneously guess a number between 0 and 100. The participant closest to $70 \%$ of the average guess wins five Euros and ties are resolved randomly. Afterwards they were randomly rematched in pairs for ten rounds to play the game shown on the left in Table 1. In COR-COR $R^{3 x 3}$ they were first matched in fixed groups of four to play the $100 \%$ version of the guessing game over five rounds. In this game all group members have to simultaneously guess a number between 0 and 100. The participant closest to the average guess wins five Euros and ties are resolved randomly. Afterwards they were randomly rematched in pairs for ten rounds to play the Coordination game shown on the right in Table 1. In IEDS-COR ${ }^{3 x 3}$ participants first played the $70 \%$ version of the guessing game over five rounds and then the $3 \times 3$ Coordination game. In COR$I E D S^{3 x 3}$, they first played the $100 \%$ version of the guessing game and then the $3 \times 3$ IEDS game. The treatment structure is summarized in Table 2.

\begin{tabular}{|l|l|l|l||l|l|l|l|}
\hline IEDS & $\mathrm{H}$ & $\mathrm{M}$ & $\mathrm{L}$ & $\mathrm{COR}$ & $\mathrm{H}$ & $\mathrm{M}$ & $\mathrm{L}$ \\
\hline $\mathrm{H}$ & 10,10 & 8,12 & 16,8 & $\mathrm{H}$ & 10,10 & 8,5 & 6,3 \\
\hline $\mathrm{M}$ & 12,8 & 10,10 & 6,6 & $\mathrm{M}$ & 5,8 & 12,12 & 5,3 \\
\hline $\mathrm{L}$ & 8,16 & 6,6 & 10,10 & $\mathrm{~L}$ & 3,6 & 3,5 & 15,15 \\
\hline
\end{tabular}

Table 1: Payoff Matrices 3x3 games.

Written instructions were distributed at the beginning of each phase. In treatments, where a sequence of two games was played, participants knew at the start of phase 1 (guessing game) that there would be a second phase in the experiment but did not know what it would look like. ${ }^{5}$ Participants were informed that they were matched with the same group of participants as in the first phase and that they would be randomly rematched with other participants in each of the periods of the second phase. This was done in

\footnotetext{
${ }^{4}$ Psychologists have studied how different isomorphic representations of the same problem or tasks can induce different solution strategies and behavioural outcomes. This is often referred to as the "representation effect", which is closely related to "framing effects" more well known among economists (Tversky and Kahneman 1981; Chen and Holyoak, 1985; Novick 1990; Zhang and Norman 1999). In some sense, those results are bad news for game theory. In this paper we show that there are also some good news. Our participants face two different problems that are structurally the same, but very differently framed, and we show that they seem to be able to learn structural properties.

${ }^{5}$ Sample Instructions for treatment IEDS- $I E D S^{3 x 3}$ can be found in Online Supplementary Material A.
} 
order to minimize the connection between the two phases. Actions H,M,L were labeled 'High', 'Medium' and 'Low' in the experimental instructions and were labeled as shown in Table 1 on the decision screens during the experiment. This labeling was chosen in order to enable participants to draw a connection between the action sets in the two games. It enables us to study the impact of learning which is based on action labels. Table 2 shows the number of (independent) observations for each treatment. ${ }^{6}$

\begin{tabular}{|l||l|l|}
\hline & $I E D S^{3 x 3}$ & $C O R^{3 x 3}$ \\
\hline \hline No first game & $\operatorname{IEDS}^{3 x 3}(480,6)$ & $C O R^{3 x 3}(480,6)$ \\
\hline COR (100 percent) & COR- $I E D S^{3 x 3}(720,6)$ & COR-COR ${ }^{3 x 3}(720,6)$ \\
\hline IEDS (70 percent) & IEDS- $I E D S^{3 x 3}(720,6)$ & IEDS- $C O R^{3 x 3}(360,3)$ \\
\hline
\end{tabular}

Table 2: Treatments together with number of (independent) observations. For the guessing games we had twice as many matching groups (independent observations).

At the end of each round of the guessing game participants were informed about the different guesses made in their group and whether they had won or not. At the end of each round of the $3 \times 3$ game participants were told their action choice and that of their match as well as their payoff. In addition to a show up fee of 2 Euros, overall earnings were the sum of earnings from all rounds. Earnings from the first phase were directly given in Euros. Earnings from the second phase were given in ECU (experimental currency unit) and converted into Euros according to the exchange rate 1 Euro=20ECU. The experiment was conducted at the BEE-Lab at Maastricht University. It lasted between 35 (control treatments) and 60 minutes (main treatments). Participants earned on average 12,30 Euros with a minimum of 6,40 Euros and a maximum of 34,20 Euros.

\section{Results}

Guessing Games Let us start by describing behavior in the guessing games. Figures (1) and (2) illustrate the distribution of guesses in the $70 \%$ version and the $100 \%$ version of the game, respectively. While in the $70 \%$ version guesses are decreasing over time, there is no such time trend in the $100 \%$ version, where most guesses are concentrated around 40-50 (see Table 3).

In the $70 \%$ version subjects' behavior seems to converge over time towards the unique Nash equilibrium where everyone guesses 0 . The modal guess is 10 . By period 5 more than $85 \%$ of guesses are below 12 . This is consistent with typical evidence from this game (Nagel, 1995). In the Keynes guessing game (100\% version) every profile where all four players choose the same number is a Nash equilibrium. The focal Nash equilibrium seems to be to choose 50, which is the amount where most initial guesses are concentrated. In fact in the Keynes version $75 \%$ of guesses are in the interval [40,60] already in period 1 and the modal guess is 50. In Online Supplementary Material D we provide graphs separately for each of the 54 matching groups (Figures D.2 - D.4). Those graphs show that also in each matching group (except for one group in COR-COR ${ }^{3 x 3}$ ) guesses converge to about 50 in the coordination version and to about 10 in the IEDS version.

\footnotetext{
${ }^{6}$ In Online Supplementary Material C we report results from additional treatments with a pure Coordination game as the $3 \times 3$ game. In this game coordination was near perfect in all treatments and reached 100 percent in the control group that only played the $3 \times 3$ game.
} 


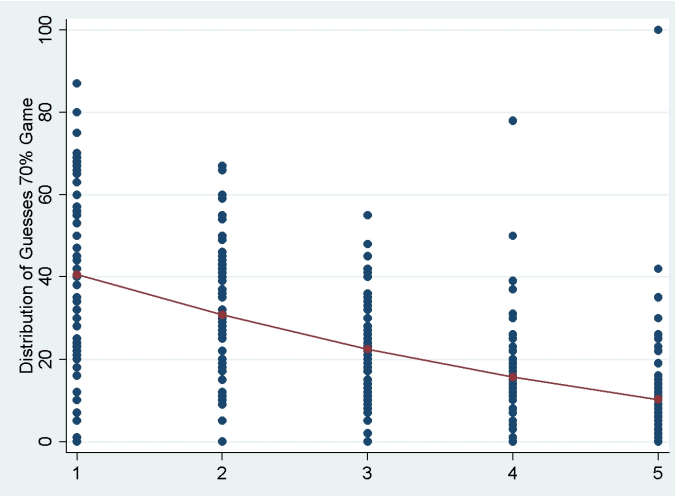

(a) IEDS Guessing Game

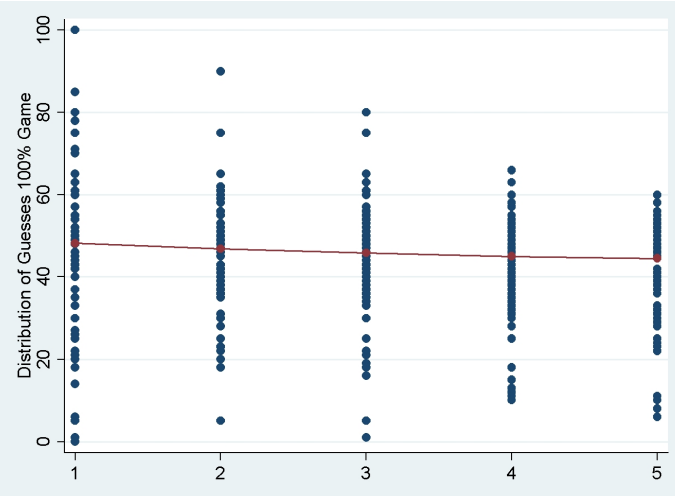

(b) Coordination Guessing Game

Figure 1: Distribution of Guesses 'IEDS guessing game' (70\% version) and 'Keynes guessing game' (100\% version). The dots depict actual guesses (data) while the line shows the guesses predicted by a linear OLS regression.

\begin{tabular}{l|ll} 
guess & $70 \%$ version & $100 \%$ version \\
\hline constant & $51.738^{* * *}$ & $49.666^{* * *}$ \\
& $(2.697)$ & $(1.492)$ \\
period & $-11.962^{* * *}$ & -1.638 \\
& $(1.497)$ & $(1.103)$ \\
period*period & $0.7336^{* * *}$ & 0.121 \\
& $(0.2243)$ & $(0.130)$ \\
\hline \hline$\rho$ & 0.3624 & 0.5164
\end{tabular}

Table 3: Random Effects OLS regression of Guesses on period. Standard errors clustered by matching group. 480 (600) observations, 96 (120) individuals, 24 (30) clusters ${ }^{* * *} 1 \%,{ }^{* *} 5 \%,{ }^{*} 10 \%$. No stars means not significant at the 10 percent level.

Based on these results we can conjecture what conventional learning models would predict regarding spillovers. ${ }^{7}$ Outcome based models, such as reinforcement learning, would predict that agents, if there are learning spillovers at all, are more likely to continue playing actions that were previously successful, i.e. make "L" choices more often after playing the IEDS guessing game and "M" choices more often after the playing the coordination version of the guessing game. Belief-based learning models, such as myopic best response learning would predict more best responses to the belief that others choose L (M) after the IEDS game (coordination game).

3x3 games We ask whether participants manage to reach the unique Nash equilibrium in the IEDS game and whether and how fast they manage to coordinate in the Coordination games. Figure 2(a) illustrates the share of M-choices (i.e. Nash choices) over time in the three IEDS treatments. The first thing to notice is that while participants seem to learn the Nash equilibrium in treatments $I E D S^{3 x 3}$ and IEDS- $I E D S^{3 x 3}$, there is no convergence in treatment COR-IED $S^{3 x 3}$. Figure 2(b) shows the analogous graph for the coordination game. Here learning seems worse in IEDS- $C O R^{3 x 3}$ compared to $C O R^{3 x 3}$ or COR-COR ${ }^{3 x 3}$.

\footnotetext{
${ }^{7}$ We discuss these models in detail in Online Supplementary Material B.
} 


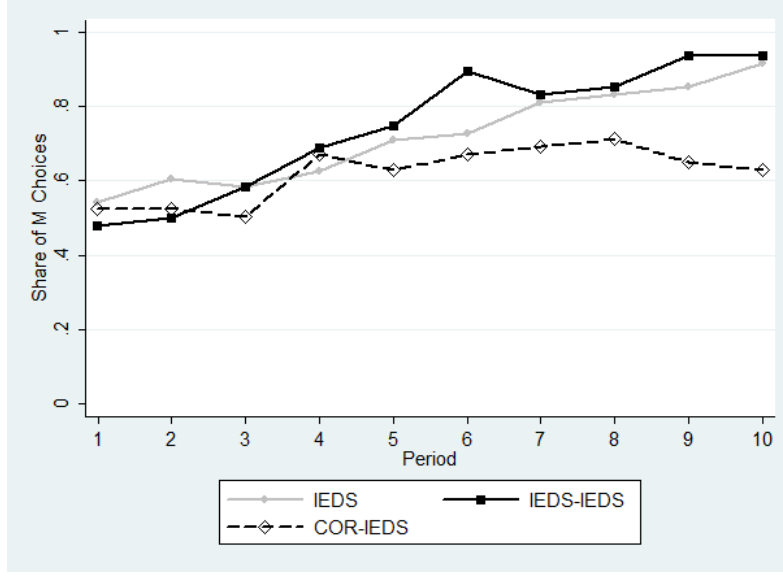

(a) IEDS Game

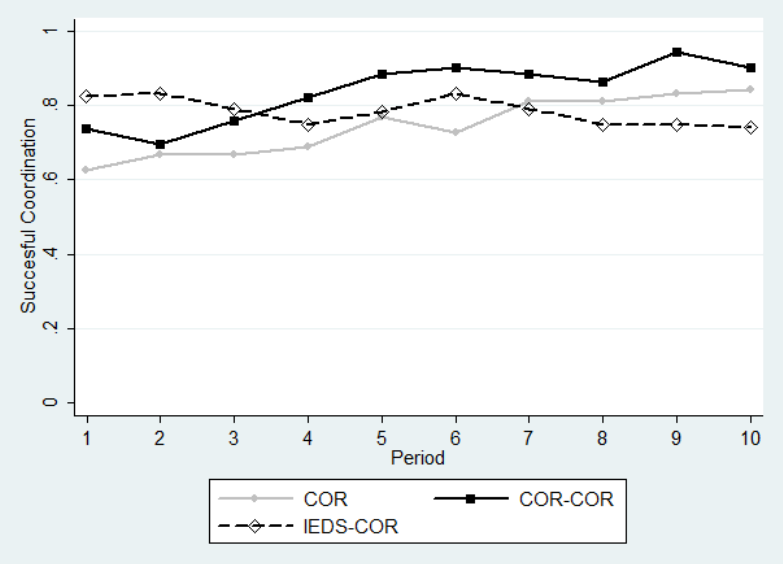

(b) Coordination Game

Figure 2: Share of M Choices over time in the 3x3 IEDS game. Rate of Successful Coordination over time in the $3 \times 3$ Coordination Game.

To understand the statistical significance of these observations, we ran logit regressions to explain the frequency of M-choices in the IEDS game (the frequency of successful coordination in the $3 \times 3$ coordination game) through the time period, treatment dummies as well as interaction terms. The period variable counts from $6, \ldots 15$ and time period in the control treatments (baseline) is normalized to this count. The results are presented in Table $4 .^{8}$

\begin{tabular}{l|ll} 
& $\left(\operatorname{IEDS}^{3 x 3}\right)$ & $\left(C O R^{3 x 3}\right)$ \\
\hline constant & $-0.1886^{* *}$ & $0.4143^{* *}$ \\
& $(0.0910)$ & $(0.2170)$ \\
similar & $-2.0137^{*}$ & -0.6095 \\
& $(0.5604)$ & $(0.6787)$ \\
dissimilar & 0.2065 & $1.5448^{* *}$ \\
period & $(0.3602)$ & $(0.5377)$ \\
& $0.2228^{* * *}$ & $0.1195^{* *}$ \\
period X similar & $(0.0682)$ & $(0.0507)$ \\
period X dissimilar & $0.1093^{* *}$ & 0.0300 \\
& $(0.0628)$ & $(0.0959)$ \\
\hline \hline$N$ & $-0.1328^{* * *}$ & $-0.1734^{* * *}$ \\
groups & $(0.0346)$ & $(0.0540)$ \\
\hline
\end{tabular}

Table 4: Random Effects Logit Regression (standard errors clustered at matching group level). ${ }^{* * *} 1 \%,{ }^{* *} 5 \%,{ }^{*} 10 \%$. $\left(\left(\operatorname{Pr}>\mathcal{X}^{2}\right)<0.0001\right)$.

The results of the regression confirm our intuition derived from Figure 2(a). The positive coefficient on the variable 'period' indicates that over time people learn to play the equilibrium in the control treatments

\footnotetext{
${ }^{8}$ In Supplementary Material D we also report the results of multinomial logit regressions on the choice between $\mathrm{H}, \mathrm{M}$ and L. Results are qualitatively robust.
} 
$I E D S^{3 x 3}\left(C O R^{3 x 3}\right)$. There are less $M$-choices in IEDS- $I E D S^{3 x 3}$ compared to $I E D S^{3 x 3}$ initially. The positive coefficient on periodXsimilar shows, however, that there is faster learning in IEDS- $I E D S^{3 x 3}$ compared to $I E D S^{3 x 3}$, i.e. more M-choices over time. ${ }^{9}$ On the other hand the negative coefficient on periodXdissimilar shows that convergence to equilibrium is worse whenever a sequence of structurally dissimilar games is played.

We ask next if we can detect any evidence for the importance of action labels, possibly in the first rounds of play for the $3 \times 3$ IEDS game. Table 5 shows the percentages of $\mathrm{M}$ and $\mathrm{L}$ choices in the first period of the $3 \times 3$ game.

\begin{tabular}{|llll|llll|}
\hline & $\mathrm{L}$ & $\mathrm{M}$ & $\mathrm{H}$ & & $\mathrm{L}$ & $\mathrm{M}$ & $\mathrm{H}$ \\
\hline$I E D S^{3 x 3}$ & 0.04 & 0.54 & 0.42 & $C O R^{3 x 3}$ & 0.62 & 0.04 & 0.34 \\
\hline IEDS- $I E D S^{3 x 3}$ & 0.06 & 0.48 & 0.46 & ${\text { COR- } C O R^{3 x 3}}^{3}$ & 0.69 & $\mathbf{0 . 1 7}$ & 0.14 \\
\hline COR- $I E D S^{3 x 3}$ & 0.06 & $\mathbf{0 . 6 3}$ & 0.31 & ${\text { IEDS- } C O R^{3 x 3}}$ & $\mathbf{0 . 8 8}$ & 0.00 & 0.12 \\
\hline
\end{tabular}

Table 5: Action Labels - M and L choices in the first period of the 3x3 games.

In the IEDS game, there are few differences. There are more M-choices in COR-IEDS ${ }^{3 x 3}$ than in $\operatorname{IED} S^{3 x 3}$, which is intuitive since players coordinate on "medium" actions in the guessing game. However, a Mann-Whitney test shows that the distribution of choices in the first round does not differ pairwise across any two treatments $(p>0.1429)$, irrespective of whether we use individuals or matching groups as independent units of independent observation. In the coordination game, participants seem to use the modal outcome of the guessing game as a coordination device for the coordination game in treatment COR-COR ${ }^{3 x 3}$. There are more $\mathrm{M}$ choices in the first period of the game in COR-COR ${ }^{3 x 3}$ compared to $C O R^{3 x 3}$ (Mann-Whitney, $p=0.0046$ using individuals as independent unit of observation). ${ }^{10}$ There are also more L choices in IEDS-COR $R^{3 x 3}$ compared to $C O R^{3 x 3}$ (Mann-Whitney, $p=0.0289$ ). Figure D.1 in the Supplementary Material illustrates that agents with very low guesses in Period 5 (in treatment COR$C O R^{3 x 3}$ do not choose $\mathrm{M}$ in period 6 . In fact the minimal guess in Period 5 out of those that choose $\mathrm{M}$ in period 6 is 22 and the maximal guess 56 .

\section{Summary, Discussion and Conclusions}

We conducted an experiment to study learning spillovers across equivalence classes of "structurally similar" games. We found that playing a structurally different game hurts convergence to Nash equilibrium, while playing a structurally similar game leads to better (faster) convergence in the second game. This is despite the fact that a simple transfer of successful actions from the guessing games to the $3 \times 3$ games would induce the opposite effect. There is one difference, though, in the impact of extrapolation on equilibrium play for games which can be solved through deliberative reasoning, such as dominance solvable games, and games for which some intuition is also needed in order to converge to a Nash equilibrium, such as coordination games. While structural similarity is important for both, in coordination games there is an additional effect. In

\footnotetext{
${ }^{9}$ The size of the coefficients implies that starting from period 10 approximately (i.e. the 5th period of the $3 \times 3$ IEDS game) there are more $M$ - choices in IEDS- $I E D S^{3 x 3}$ compared to $I E D S^{3 x 3}$.

${ }^{10}$ Since we restrict attention to the first period of the $3 \times 3$ game each individual is indeed an independent observation in treatments $C O R^{3 x 3}$. In all other treatments the 6(12) matching groups from the guessing games are the independent units of observation. However we did not want to compare a binary variable in one treatment with matching group averages from another treatment. Hence we used individuals as independent unit of observation for all the Mann-Whitney tests reported here.
} 
these games players try to use the modal behavior in the first game as a coordination device for the second game.

The results we observed cannot be explained by conventional outcome based or belief based learning models, where agents learn about successful actions (e.g. reinforcement learning) or learn about their opponents' behaviour and try to best respond to it.

One possible explanation is that participants learn about the structural properties of the guessing games and then try to apply this knowledge afterwards, leading to better convergence in IEDS-IED $S^{3 \times 3}$ and worse convergence in COR-IED $S^{3 x 3}$. On the other hand, playing a coordination game where intuition (or some 'gut-feeling') is needed to reach a Nash equilibrium, may bring participants into the wrong 'mode' of play when afterwards playing a game which is solvable through IEDS. ${ }^{11}$

These results can be very helpful to solve coordination problems, to make predictions about behaviour under new institutions or laws or for the design of organizations. Understanding how knowledge is transferred between games and how this depends on the type of strategic situation faced can also inform theoretical models of learning across games and categorization. Most of the current literature on categorization or learning across games focuses on categorization along action labels. ${ }^{12}$ Understanding structural learning and improved learning via extrapolation of strategic context seems harder to model and to understand. There is large scope for future research in this area.

\section{References}

[1] Camerer, C., T. Ho and K. Weigelt (1998), Iterated Dominance and Iterated Best Response in Experimental "p-Beauty Contests", American Economic Review, 88(4), 947-969.

[2] Camerer, C., T-H. Ho, and J-K Chong (2004), A cognitive hierarchy model of Games, The Quarterly Journal of Economics, 861-898.

[3] Cason, T. and A. Savikhin and R. Sheremata (2012), Behavioral Spillovers in Coordination Games, European Economic Review 56, 233-245.

[4] Cheng, P.W. and Holyoak, K. J. (1985), Pragmatic reasoning schemas. Cognitive Psychology, 17, 391416.

[5] Chong, J-K., C. Camerer and T. Ho (2006), A Learning-based Model of Repeated Games with Incomplete Information, Games and Economic Behavior, 55(2), 340-371

[6] Cooper, D. J. and J. H. Kagel (2003), Lessons Learned: Generalizing Learning across Games, American Economic Review, 93(2), 202-207.

[7] Germano, F. (2006), On some geometry and equivalence classes of normal form games, International Journal of Game Theory 34: 561-581.

[8] Gilboa, I. and D. Schmeidler (1995), Case-Based Decision Theory, The Quarterly Journal of Economics, 110(3), 605-639.

\footnotetext{
${ }^{11}$ In support of this explanation, Kuo et al. (2009) find evidence that two different parts of the brain may be used to play these different classes of games.

${ }^{12}$ See e.g. Jehiel (2005), Steiner and Stewart (2008), Mengel (2012) or Mohlin (2014).
} 
[9] Grimm, V. and F. Mengel (2012), An Experiment on Learning in a Multiple Games Environment, Journal of Economic Theory 147 (6), 2220-2259.

[10] Haruvy, E. and D. Stahl (2009), Learning Transference between dissimilar symmetric normal-form games, working paper.

[11] Heinemann, F., R. Nagel and P. Ockenfels (2009), Measuring Strategic Uncertainty in Coordination Games, Review of Economic Studies 76(1), 181-221.

[12] Huck, S., P. Jehiel and T. Rutter (2011), Learning Spillover and Analogy Based Expectations: A Multi Game Experiment, Games and Economic Behavior 71, 351-365.

[13] Jehiel, P.(2005), Analogy-based expectation equilibrium, Journal of Economic Theory 123, 81-104.

[14] Kahneman, D. and A. Tversky (1981), The Framing of decisions and the psychology of choice, Science 211 (4481), 453-458.

[15] Keynes, J.M. (1936), The general theory of interest, employment and money. London: Macmillan.

[16] Kuo, W.J., T. Sjoestrom, Y.P. Chen, Y.H. Wang and C.Y. Huang (2009), Intuition and Deliberation: Two Systems for Strategizing in the Brain, Science 324, 519-522.

[17] Mengel, F. (2012), Learning Across Games, Games and Economic Behavior 74(2), 601-619.

[18] Mohlin, E. (2014), Optimal Categorization, Journal of Economic Theory 152, 356-381.

[19] Nagel, R. (1995), Unraveling in Guessing Games: An Experimental Study, American Economic Review 85, 1313-1326.

[20] Novick, L. (1990), Representational transfer in problem solving, Psychological Science 1, 128-132.

[21] Rankin, F., J. B. van Huyck and R. Battalio (2000), Strategic Similarity and Emergent Conventions: Evidence from Similar Stag Hunt Games, Games and Economic Behavior 32, 315-337.

[22] Rapoport, A., A. Seale and E. Winter (2000), An experimental study of coordination and learning in iterated two-market entry games, Economic Theory 16 (3), 1432-1479.

[23] Steiner, J. and C. Stewart (2008), Contagion Through Learning, Theoretical Economics 3, 431-458.

[24] Zhang, J. and Norman, D. A. (1994). Representations in distributed cognitive tasks. Cognitive Science, $18,87-122$. 